\title{
GASTROENTEROLOGY
}

\section{Costs and outcomes of Lynch syndrome screening in the Australian colorectal cancer population}

\author{
Dayna R Cenin, ${ }^{*},+, \neq$ (D) Steffie K Naber, ${ }^{*}$ Iris Lansdorp-Vogelaar, ${ }^{*}$ Mark A Jenkins, ${ }^{\S}$ Daniel D Buchanan,,${ }^{\S, 1, * *,+\dagger}$ (iD \\ David B Preen, ${ }^{\ddagger}$ Hooi C Ee ${ }^{\ddagger \ddagger}$ and Peter O'Leary ${ }^{\dagger, \S \S, १ \uparrow}$ \\ ${ }^{*}$ Department of Public Health, Erasmus Medical Center, Rotterdam, The Netherlands; and ${ }^{\dagger}$ Faculty of Health Sciences, Curtin University, ${ }^{\ddagger}$ Centre for Health \\ Services Research, School of Population and Global Health, ${ }^{\S \S}$ School of Women's and Infants' Health, The University of Western Australia, and \\ ${ }^{\ddagger \neq}$ Department of Gastroenterology, Sir Charles Gairdner Hospital, Perth, "19lPathWest Laboratory Medicine, QE2 Medical Centre, Nedlands, Western \\ Australia, ${ }^{\S}$ Centre for Epidemiology and Biostatistics, Melbourne School of Population and Global Health, University of Melbourne, Melbourne, " Colorectal \\ Oncogenomics Group, Genetic Epidemiology Laboratory, Department of Pathology, University of Melbourne, **Genetic Medicine and Familial Cancer \\ Centre, The Royal Melbourne Hospital, and ${ }^{\dagger+}$ Victorian Comprehensive Cancer Centre, University of Melbourne Centre for Cancer Research, Parkville, \\ Victoria, Australia
}

\section{Key words}

BRAF V600E, colorectal cancer, costeffectiveness, Lynch syndrome, mismatch repair, MLH1 methylation, screening

Accepted for publication 24 March 2018

\section{Correspondence}

Dayna R Cenin, Department of Public Health, Erasmus Medical Center, P.O. Box 2040, Rotterdam 3000 CA, The Netherlands.

Email: d.cenin@erasmusmc.nl

Declaration of conflict of interest: The authors declare no conflicts of interest. M. A. J. is an NHMRC Senior Research Fellow. D. D. B. is a University of Melbourne Research at Melbourne Accelerator Program (R@MAP) Senior Research Fellow and NHMRC R.D. Wright Career Development Fellow.

Financial support: D. R. C., D. B. P., H. C. E., and P. O. L. are funded by the Cancer Council Western Australia Capacity and Collaboration Building Grant. The colorectal cancer data in this analysis were supported by the grant UM1 CA167551 from the National Cancer Institute and through cooperative agreements with Australasian Colorectal Cancer Family Registry (U01 CA074778 and U01/U24 CA097735) and were conducted under Colon-CFR approval CAU-0312-01. The Melbourne Collaborative Cohort Study for colorectal cancer was funded by the NHMRC project grant 509348 (PI-Dallas English) "Risk Factors for Molecular Subtypes of Colorectal Cancer."

\begin{abstract}
Background and Aim: Individuals with Lynch syndrome (LS) are at increased risk of LSrelated cancers including colorectal cancer (CRC). CRC tumor screening for mismatch repair (MMR) deficiency is recommended in Australia to identify LS, although its costeffectiveness has not been assessed. We aim to determine the cost-effectiveness of screening individuals with CRC for LS at different age-at-diagnosis thresholds.

Methods: We developed a decision analysis model to estimate yield and costs of LS screening. Age-specific probabilities of LS diagnosis were based on Australian data. Two CRC tumor screening pathways were assessed (MMR immunohistochemistry followed by MLH1 methylation (MLH1-Pathway) or BRAF V600E testing (BRAF-Pathway) if $M L H 1$ expression was lost) for four age-at-diagnosis thresholds-screening $<50$, screening $<60$, screening $<70$, and universal screening.

Results: Per 1000 CRC cases, screening $<50$ identified 5.2 LS cases and cost $\$ A 7041$ per case detected in the MLHI-Pathway. Screening $<60$ increased detection by 1.5 cases for an incremental cost of \$A25 177 per additional case detected. Screening $<70$ detected 1.6 additional cases at an incremental cost of \$A40 278 per additional case detected. Compared with screening $<70$, universal screening detected no additional LS cases but cost \$A158 724 extra. The BRAF-Pathway identified the same number of LS cases for higher costs.

Conclusions: The MLH1-Pathway is more cost-effective than BRAF-Pathway for all ageat-diagnosis thresholds. MMR immunohistochemistry tumor screening in individuals diagnosed with CRC aged $<70$ years resulted in higher LS case detection at a reasonable cost. Further research into the yield of LS screening in CRC patients $\geq 70$ years is needed to determine if universal screening is justified.
\end{abstract}

\section{Introduction}

Colorectal cancer (CRC) is a leading cause of cancer incidence and mortality in Australia. ${ }^{1}$ While diagnoses are predominantly made in those at older ages, certain groups are at increased risk of early-onset CRC, largely as a result of inherited genetic mutations. ${ }^{2}$ Lynch syndrome (LS), an autosomal dominant condition, is a well-known genetic syndrome that increases risk of early- 
onset CRC (average age at diagnosis is 42 years for men and 47 years for women ${ }^{3}$ ). Caused by a germline mutation in one of the DNA mismatch repair (MMR) genes (MLH1, MSH2, MSH6, or PMS2), LS is characterized by tumors that develop with high levels of microsatellite instability (MSI) and loss of expression of one or more of the MMR proteins, collectively referred to as tumor MMR deficiency.

Lynch syndrome is estimated to cause $1-3 \%$ of all CRC cases ${ }^{4}$ with carriers experiencing accelerated carcinogenesis and an increased lifetime risk for CRC $\left(10-47 \%\right.$ by age 70 years ${ }^{5-7}$ compared with $4-5 \%^{8}$ in the general population) as well as predisposing individuals to other cancers. ${ }^{9,10}$ A diagnosis of LS aids clinical decision-making, including more extensive surgery and highly intensive long-term surveillance, which impacts patient outcomes. ${ }^{4}$ Furthermore, a diagnosis permits cascade testing of atrisk family members to determine LS carrier status, thus enabling the commencement of intensive surveillance, which has been shown to lead to a reduction in LS-related cancer incidence and mortality. ${ }^{11-15}$

Historically, LS testing has been guided using the Amsterdam or revised Bethesda criteria, both of which rely on obtaining an accurate family history ${ }^{12}$ but have limited sensitivity and specificity for LS detection and are poorly implemented in routine clinical practice. ${ }^{4,16,17}$ More recently, screening for LS has begun with tumor testing for MMR deficiency, prior to proceeding to germline MMR gene testing. ${ }^{17-19}$ However, as MMR deficiency can also be caused by sporadic somatic hypermethylation of the MLH1 gene promoter, tumors showing loss of MLH1/PMS2 protein expression require further testing (with either somatic $M L H 1$ methylation testing or $B R A F$ V600E somatic mutation testing). If LS is still suspected after these tumor tests, genetic testing is offered in association with genetic counseling.

Within Australia, there is no national policy for LS screening; however, the National Health and Medical Research Council recently recommended universal screening, ${ }^{20}$ as a means of increasing identification of carriers and their at-risk relatives. While this recommendation is in line with other juristrictions, ${ }^{4,21}$ no costeffectiveness analyses have been conducted in the Australian setting and therefore the optimal screening strategy remains unclear.

We aimed to determine the cost-effectiveness of CRC tumor screening to identify LS at different age-at-diagnosis thresholds for two alternative tumor screening pathways using data from the Australian setting.

\section{Methods}

Overview. We developed a decision analysis model to simulate LS screening in individuals with CRC to estimate the annual yield and costs associated with identifying LS this population. For tumors exhibiting loss of MLH1/PMS2 expression by MMR immunohistochemistry (IHC), we tested two alternative pathways based on the follow-up tumor test (MLH1 methylation test or a BRAF V600E mutation test). The primary focus was to determine how yield and cost would vary for each pathway by age-at-diagnosis and compare the incremental differences within and between the pathways.

Data. Model parameters were based on two Australian research studies, the Australasian Colorectal Cancer Family Registry and the Melbourne Collaborative Cohort Study, which have been systematically characterized for LS. Detailed information about the recruitment strategy and tumor testing for these studies has been previously reported. ${ }^{18}$ In brief, the Australasian Colorectal Cancer Family Registry recruited population-based incident CRC cases of individuals aged $18-59$ years (eligible cases $n=813$ ) between 1997 and 2007. The Melbourne Collaborative Cohort Study is an Australian cohort study of 41513 Melbourne residents recruited during 1990-1994 with age range at recruitment of 27-80 years. Data from 826 CRC cases diagnosed from recruitment until 2010 and aged 41-86 years at diagnosis were used for this analysis.

Colorectal cancer tumor samples from both studies were tested for MMR protein expression using IHC. Tumors showing MMR deficiency underwent germline testing to identify a MMR gene mutation and confirm LS diagnosis. For tumors demonstrating loss of $M L H 1 / \mathrm{PMS} 2$ expression by IHC, testing for tumor $M L H 1$ promoter hypermethylation and $B R A F$ V600E somatic mutation were performed, and only those cases with no evidence of somatic $M L H 1$ methylation or BRAF wild-type underwent germline testing of $M L H 1$ gene.

Decision analysis model. Using TreeAge Pro 2016 (Williamstown, Massachusetts), we developed a decision analysis model to simulate LS screening. For tumors exhibiting loss of MLH1/PMS2 expression by MMR IHC, we assessed two screening pathways for identifying LS based on follow-up tumor testing. In the first model (MLH1-Pathway), IHC was followed by somatic MLH1 methylation testing (Fig. 1), while in the second model (BRAF-Pathway), IHC was followed by BRAF V600E mutation testing (Fig. 2). For each pathway, we simulated 1000 CRC cases and assumed $100 \%$ participation in tumor and genetic testing at all stages. Once a diagnosis of CRC has been made, eligible individuals entered the LS screening pathway and progressed based on age-specific probabilities (Figs 1, 2). Costs are applied at appropriate time points along the pathway, such as when a test is conducted or when genetic counseling would be initiated.

Screening scenarios. For this analysis, we used empirical data $^{18}$ to assess four age-at-diagnosis scenarios: screening $<50$, screening $<60$, screening $<70$, and universal screening. In the reference scenario, screening $<50$, screening was restricted to CRC diagnoses occurring before the age of 50 years. Screening $<60$ expanded tumor screening to include those aged 50-59 years, and screening $<70$ is a further expansion to include cases aged 6069 years. The universal scenario included screening of all incident CRC diagnoses regardless of age. The probability of meeting the LS screening eligibility criteria for the age-restricted scenarios was based on Australian CRC incidence data from 2008 to $2012 .{ }^{8}$

Cost assumptions. The cost of MMR IHC was provided by The Royal College of Pathologists of Australasia Benchmarking in Pathology Quality Assurance Program (St. Leonards, NSW) (2013) results (Dr Tony Badrick, pers. comm.). For the MLH1 methylation testing, cost data were provided by PathWest Laboratory Medicine, Nedlands, the sole government pathology service for Western Australia (Dr Benhur Amanuel, pers. comm.). The 


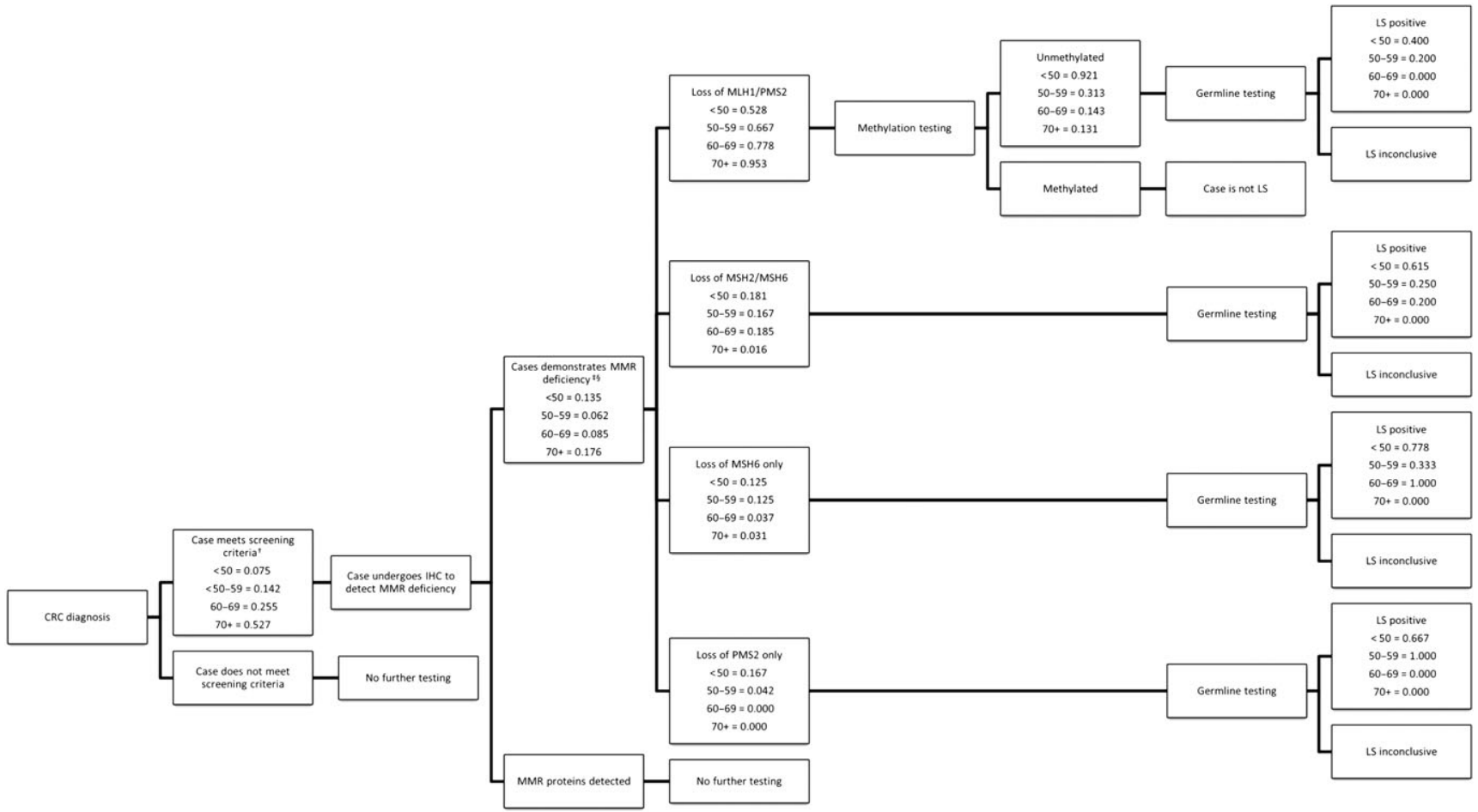

Figure 1 MLH1-Pathway with age-specific probabilities of progressing through the Lynch syndrome (LS) screening pathway. ${ }^{\dagger}$ Probability of meeting inclusion criteria in the age-restricted scenarios is based on the age distribution of colorectal cancer (CRC) incidence data from 2008 to $2012 .{ }^{8}{ }^{\ddagger} \mathrm{MMR}$ deficiency is determined by testing with IHC and is defined as loss of MMR expression in one or more of the four MMR genes (MLH1, PMS2, MSH2, and MSH6). ${ }^{\S}$ Progression through the pathway is based on probabilities derived from Buchanan et al. ${ }^{18}$ These probabilities differ slightly as we considered LS cases that did not show MMR deficiency with IHC to be missed cases (three cases in screening $<60$ and screening $<70$ and four cases in universal). In addition, one LS case was excluded from the probabilities in our analysis because although the case showed PMS2 loss, genetic testing identified an MLH1 mutation, and this could not be factored into the model. Using screening $<50$ as the example, $7.6 \%$ of all CRC cases were eligible for testing with IHC to determine MMR deficiency status and 13.5\% were MMR deficient. Of these, $52.8 \%$ had loss of MLH1/PMS2, 18.1\% had loss of MSH2/MSH6, $12.5 \%$ had loss of MSH6 only, and 16.7\% had loss of PMS2 only. Of the tumors with of MHL1/PMS2, 92.1\% were unmethylated and went on for germline testing. LS was confirmed in 66.7\% of CRC cases demonstrating MLH1/PMS2 loss (excluding MLH1-methylated CRCs), $61.5 \%$ of the cases demonstrating MSH2/MSH6 loss, $77.8 \%$ of the cases demonstrating MSH6 loss, and $66.7 \%$ of the cases demonstrating PMS2 only. CRC, colorectal cancer; IHC, immunohistochemistry; LS, Lynch syndrome; MMR, mismatch repair; < 60, age-specific probabilities for CRC cases aged under 60 years; 60-69, age-specific probabilities for CRC cases aged between 60 and 69 years; 70+, age-specific probabilities for CRC cases aged over 70 years.

cost of $B R A F$ V600E testing was taken from MBS Online ${ }^{22}$ (Table 1). Germline testing costs were provided by the Department of Diagnostic Genomics, PathWest Laboratory Medicine, Nedlands, the primary laboratory for genetic testing in Western Australia (Dr Karen Carpenter, pers. comm.). The costs for genetic counseling were obtained from primary sources at Genetic Services of Western Australia (Subiaco, WA), including the Business Unit and genetic counselors (Anne Hawkins and Cassandra Nichols, pers. comm.).

All costs are presented in 2016 Australian dollars, and as they are incurred in a single year, no discounting is required.

Outcomes. For each screening pathway, our decision analysis model estimated the annual yield and costs of identifying LS in the four age-restricted scenarios per 1000 CRC cases.

Sensitivity analyses. To evaluate the robustness of our model outcomes, we conducted a number if univariate analyses.
Firstly, we assessed the uncertainty of the diagnostic accuracy by calculating the $95 \%$ confidence intervals around the probability of being diagnosed with LS after demonstrating MMR deficiency using the Wilson confidence interval. This provided lower and upper confidence limits of yield and costs of LS screening in the CRC population.

Furthermore, as no cases of LS were diagnosed in CRC patients aged $\geq 70$ years in our data set, we performed an analysis of the MLH1-Pathway using age-specific probabilities derived from Hampel et al. ${ }^{30}$ to assess the impact of identifying LS cases in this age group. Unfortunately, similar data were not available from this research to assess the $B R A F$-Pathway.

Finally, we reduced acceptance of genetic counseling to $92.5 \%^{23}$ and varied the acceptance of germline testing to $81 \%{ }^{17}$ and $90 \%{ }^{23}$ to assess the impact of these variables on yield and costs of LS screening.

For all sensitivity analyses, we also explored the effect of varying costs parameters by assuming a $50 \%$ reduction and a twofold 


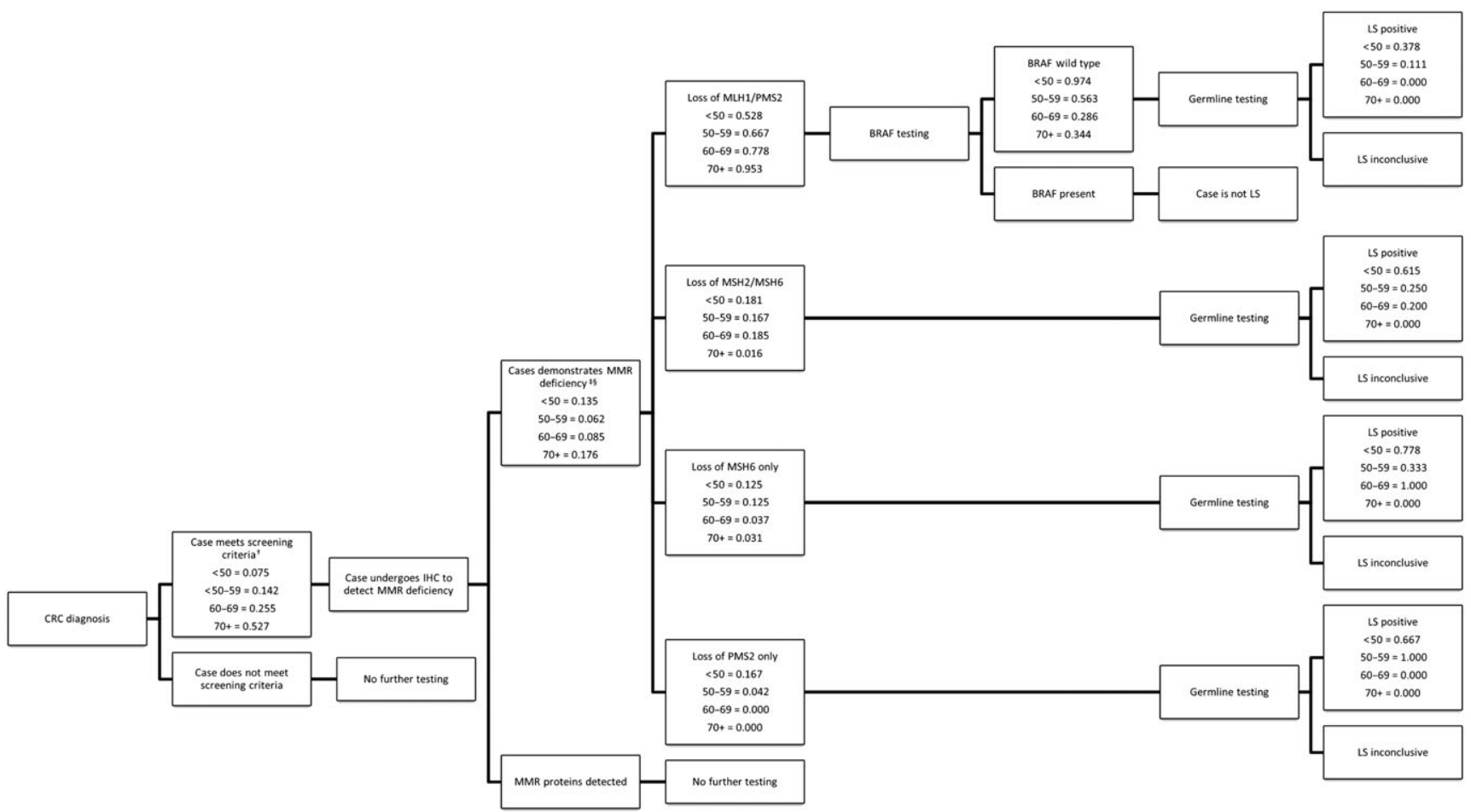

Figure 2 BRAF-Pathway with age-specific probabilities of progressing through the Lynch syndrome (LS) screening pathway. ${ }^{\dagger}$ Probability of meeting inclusion criteria in the age-restricted scenarios is based on the age distribution of colorectal cancer (CRC) incidence data from 2008 to $2012 .{ }^{7}{ }^{\ddagger} \mathrm{MMR}$ deficiency is determined by testing with IHC and is defined as loss of MMR expression in one or more of the four MMR genes (MLH1, PMS2, MSH2, and MSH6). ${ }^{\S}$ Progression through the pathway is based on probabilities derived from Buchanan et al. ${ }^{18}$ These probabilities differ slightly as we considered LS cases that did not show MMR deficiency with IHC to be missed cases (three cases in screening $<60$ and screening $<70$ and four cases in universal). In addition, one LS case was excluded from the probabilities in our analysis because although the case showed PMS2 loss, genetic testing identified an MLH1 mutation, and this could not be factored into the model. Using screening $<50$ as the example, $7.6 \%$ of all CRC cases were eligible for testing with IHC to determine MMR deficiency status and 13.5\% were MMR deficient. Of these, 52.8\% had loss of MLH1/PMS2, 18.1\% had loss of MSH2/MSH6, $12.5 \%$ had loss of MSH6 only, and $16.7 \%$ had loss of PMS2 only. Of the tumors with of MHL1/PMS2, 97.4\% were BRAF wild type and went on for germline testing. LS was confirmed in 37.8\% of CRC cases demonstrating MLH1/PMS2 loss, $61.5 \%$ of the cases demonstrating MSH2/MSH6 loss, $77.8 \%$ of the cases demonstrating MSH6 loss, and 66.7\% of the cases demonstrating PMS2 only. CRC, colorectal cancer; IHC, immunohistochemistry; LS, Lynch syndrome; MMR, mismatch repair; < 60, age-specific probabilities for CRC cases aged under 60 years; 60-69, age-specific probabilities for CRC cases aged between 60 and 69 years; 70+, age-specific probabilities for CRC cases aged over 70 years.

increase of all costs (Table 1 and tables in the Supporting Information). This provided lower and upper bound cost estimates for each age cohort in the analyses.

\section{Results}

MLH1-Pathway. By restricting testing to CRC cases diagnosed $<50$ years, $76(7.6 \%)$ of the 1000 CRC cases would be tested with IHC, leading to the identification of 5.2 LS cases. Total costs for this pathway were $\$ 36864$ per 1000 CRC cases, equating to $\$ 7041$ per $\mathrm{LS}$ case diagnosed.

By expanding screening to include those aged between 50 and 59 years (screening $<60$ ), an extra 142 individuals (totaling $21.8 \%$ of total CRC patient population) would be tested with IHC to identify 1.5 additional LS cases (6.7 LS cases in total). This would cost an additional \$36 794 or \$25 177 per additional LS case diagnosed. Cost per case detected increased to \$10 999 .

With further expansion to also screen CRC cases aged between 60 and 69 years (screening $<70$ ), an additional 255 individuals (totaling $47.3 \%$ of total CRC patient population) would be tested by IHC. This identified 1.6 additional LS cases (8.3 LS cases in total), annual program cost increased to $\$ 138663$, and the cost per additional case detected was $\$ 40278$. Cost per case detected increased to $\$ 16685$.

Based on our data, universal screening would not identify any additional LS cases; however, annual program cost would increase by $\$ 158724$ to $\$ 297387$ per 1000 CRC cases. Cost per LS case detected increased to $\$ 35784$.

BRAF-Pathway. The BRAF-Pathway identified the same number of LS cases as the MLH1-Pathway at higher costs (Table 2). For example, screening $<50$ identified 5.2 LS cases per $1000 \mathrm{CRC}$ cases and cost $\$ 36462$ for the MLH1-Pathway and $\$ 37177$ in the BRAF-Pathway. Therefore, LS screening based on IHC followed by BRAF V600E is more expensive than the alternative, and the $B R A F$-Pathway is subsequently dominated in terms of cost-effectiveness. 


\begin{tabular}{|c|c|c|c|}
\hline Parameter & Cost & Source & Range $(\$ A)$ \\
\hline \multicolumn{4}{|l|}{ Molecular tests } \\
\hline Mismatch repair immunohistochemistry & 175 & Expert opinion, Dr Tony Badrick, RCPAQAP (e-mail) & $88-350^{\dagger}$ \\
\hline MLH1 methylation testing & 314 & $\begin{array}{l}\text { Expert opinion, Dr Benhur Amanuel, PathWest } \\
\text { Laboratory Medicine (e-mail) }\end{array}$ & $157-628^{\dagger}$ \\
\hline$B R A F$ V600E testing & 231 & MBS Online 22 & $115-462^{\dagger}$ \\
\hline Combined diagnostic genetic test $\mathrm{MLH} 1, \mathrm{MSH} 2$, and $\mathrm{MSH}^{\ddagger}$ & 1400 & Expert opinion, Dr Karen Carpenter, PathWest & $700-2800^{\dagger}$ \\
\hline Diagnostic genetic test $\mathrm{PMS} 2^{\S}$ & 1000 & Diagnostic Genomics (e-mail) & $500-2000^{\dagger}$ \\
\hline \multicolumn{4}{|l|}{ Genetic counseling } \\
\hline Initial session & $267^{\dagger+}$ & Expert opinion, Anne Hawkins and Cassandra Nichols, & $92-455^{\ddagger \neq}$ \\
\hline LS diagnosis & $251^{\dagger+}$ & Genetic Services of Western Australia (e-mail) & $78-438^{\ddagger \neq}$ \\
\hline LS inconclusive & $22^{\dagger \dagger}$ & & $7-36^{\ddagger \ddagger}$ \\
\hline
\end{tabular}

All costs are presented in 2016 Australian dollars.

${ }^{\dagger}$ Extrapolated range based on $50 \%$ reduction and a twofold increase.

‡Based on Illumina TruSight Cancer MPS panel (San Diego, California, USA) and two MLPA kits (MRC-Holland, Amsterdam, The Netherlands) for $\mathrm{MLH} 1, \mathrm{MSH} 2$, and MSH6.

${ }^{\S}$ Based on long-range PCR followed by Sanger sequencing and MLPA for PMS2.

"Costs for genetic counseling vary according to the complexity of the counseling provided. To calculate the cost of the genetic counseling, we first established a range of costs using the shortest and longest duration of genetic counseling and the least to most complex counseling scenarios. The average of these values was used in the analysis. Costs are divided into initial cost for genetic counseling, which includes planning and preparation for individual consultations, and follow-up costs, which vary depending on the outcome of the genetic test.

${ }^{+\dagger}$ Mean cost of providing each service.

${ }^{\ddagger \neq}$ Range based on minimum duration and complexity to maximum duration and complexity of counseling service.

LS, Lynch syndrome; MLPA, Multiple Ligation-dependent Probe Amplification; MPS, Massive Parallel Sequencing; PCR, polymerase chain reaction; RCPAQAP, Royal College of Pathologists of Australasia Quality Assurance Program.

Sensitivity analyses. When the probability of a diagnosis of LS was altered, our results changed significantly (Table S1). Although overall costs remained similar to the original analysis, in the lower bound analysis, the number of LS cases diagnosed reduced to between 3.2 and 3.8, while the cost per LS case diagnosed increased ranged from $\$ 11521$ to $\$ 79091$. The reverse was true for the upper bound analysis where the number of LS cases diagnosed increased to between 7.0 and 23.7 and cost per case detected ranged from $\$ 5350$ to $\$ 13731$. A similar pattern was seen when the age-specific probabilities derived from Hampel et $a .^{23}$ were applied to the MLH1-Pathway. Using these data, the number of LS cases diagnosed in each age restricted scenario was higher, and the cost per LS case diagnosed was lower. However, program costs remained similar to our original analysis (Table S2).

Lowering adherence to genetic counseling and germline testing reduced diagnostic yield by up to $25 \%$. This resulted in a slight reduction in total costs (5-15\% for MLH1-Pathway and 7-15\% for $B R A F$-Pathway), while the cost per LS case detected increased (8-24\% for MLH1-Pathway and $8-21 \%$ for BRAF-Pathway) (Table S3). Similar results were found when both costs and acceptance of genetic counseling and germline testing were altered.

Changes to the cost parameters affected the costs proportionally (Table 2 and tables in the Supporting Information).

\section{Discussion}

We developed a decision analysis model and used empirical data ${ }^{18}$ to determine the cost and yield of screening for LS per 1000 CRC cases. Based on our results, screening for LS using the MLH1Pathway is more cost-effective than the BRAF-Pathway. Limiting screening to CRC cases aged under 50 years in the MLHI-
Pathway would identify 5.2 LS cases per 1000 CRC cases for the overall lowest cost, with a cost per LS case detected of $\$ 7041$. Expanding this pathway to also screen individuals aged $50-59$ years (screening $<60$ ) increased diagnostic yield by $28 \%$ (1.5 cases). This was associated with a doubling of program costs, an increase in cost per LS case detected (to \$10 999) and an incremental cost of \$25 177 to detect one additional case. Screening $<70$ further increased diagnostic yield of screening with program costs increasing by $88 \%$. Cost per case detected in this scenario increased to $\$ 16685$, equating to an incremental cost per additional case detected of $\$ 40278$. Universal screening more than doubled program costs compared with screening $<70$ for no additional yield; however, this was because no LS cases were identified in this age group in our dataset. Cost per LS case detected increased to $\$ 35784$. The BRAF-Pathway identified the same number of LS cases; however, costs were higher for all age-at-diagnosis thresholds.

There remains ongoing discussion about the optimal age to stop screening for LS in the CRC-affected population, ${ }^{4,21}$ and our model, like others, ${ }^{24-27}$ demonstrates that applying age restrictions to screening criteria results in fewer LS cases being identified. This impacts patient care and has downstream effects for at-risk relatives who would not be identified, thereby diminishing the opportunity to commence interventions to reduce mortality and morbidity in this cohort. However, concerns have been raised about the feasibility of expanded screening for LS, particularly in relation to the associated costs. ${ }^{24}$ While individuals with LS are at higher risk of LS-related cancers compared with the general population, the likelihood of developing such a cancer diminishes with age. ${ }^{14}$ This suggests that, while expanding screening to include older individuals will identify more cases, the increased 


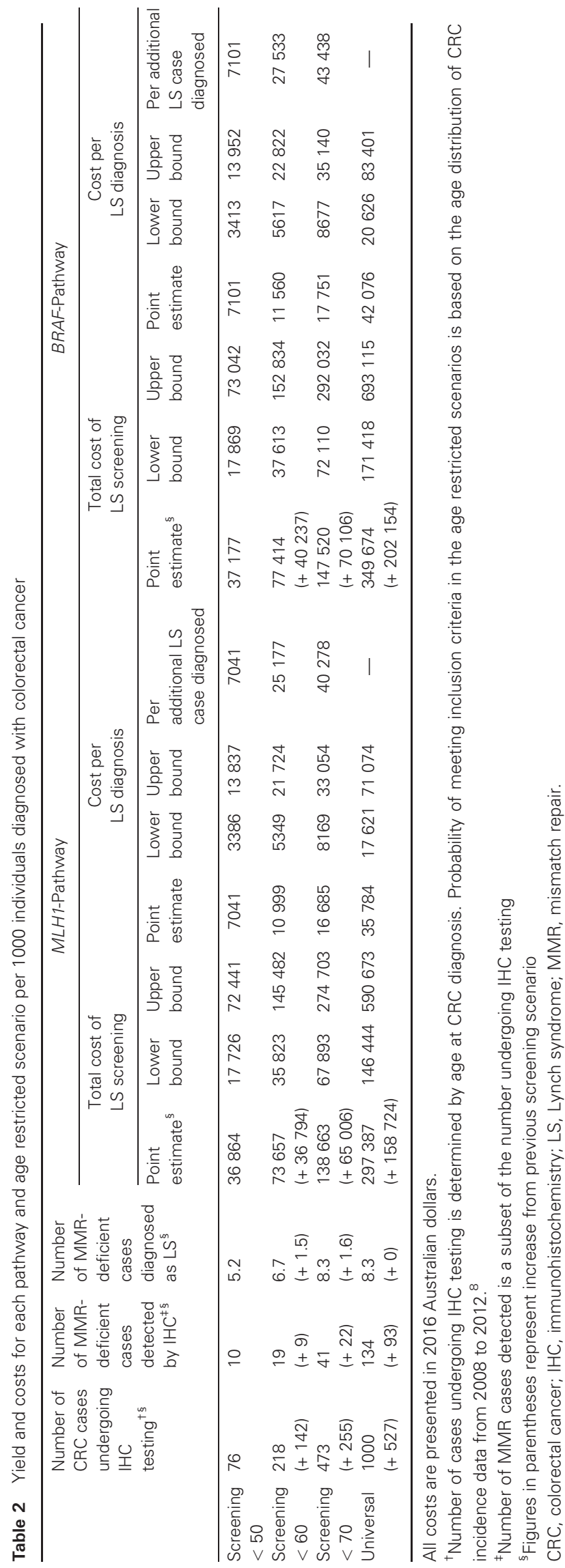

detection will likely come at the expense of efficiency. In our model, the proportion of CRC cases who demonstrate MMR protein loss by IHC and are subsequently found to be LS positive was highest in the screening $<50$ scenario ( $52 \%$ for both pathways). This congruency reduced in the expanded alternatives (ranging from $0 \%$ to $16 \%$ ), demonstrating that although screening older individuals may detect additional LS cases, it does so by conducting disproportionately greater numbers of tests which results in higher program costs. This increase in overall cost could be considerable, with our model demonstrating that universal screening cost was more than twice as much as screening $<70$ with no additional benefit in LS carrier detection.

Based on our results, there is added benefit in ensuring CRC cases $<70$ years are screened and although this requires an increase in total program cost, such an expansion could be considered a reasonable trade-off between costs and yield. ${ }^{25,27}$ While our model suggests there is no additional benefit of universal screening compared with screening $<70$, this is due to the lack of LS cases identified in this age cohort in our study population. As other investigations have confirmed the existence of CRCaffected LS mutation carriers in this age group, ${ }^{19,28}$ we conducted a sensitivity analysis where we assessed the impact of adjusting the proportion of individuals who were MMR deficient but were not confirmed with LS (Table S1). In the case of universal screening, increasing this proportion of individuals diagnosed with LS dramatically reduced the cost per LS case diagnosed (to between $\$ 14000$ and \$15 000) and the cost per additional LS diagnosis (approximately $\$ 25000)$. In a second analysis, we applied the agespecific probabilities of a US study evaluating the MLH1Pathway. ${ }^{30}$ Using these probabilities, we found similar overall program costs for universal screening with a cost per additional LS case detected of approximately $\$ 50000$ (Table S2). These results indicate that while there is potential benefit in screening CRC cases $\geq 70$ years in terms of yield, cost-effectiveness of such an expansion will be significantly impacted by the proportion of LS cases in this age cohort. Further studies with larger samples are needed to enable more precise estimates.

Although screening for LS has shown to be cost-effective, consensus of the optimal strategy is yet to be achieved. To our knowledge, few studies have presented results based on age-restricted inclusion criteria for the two pathways we investigated. One analysis investigating the $B R A F$-Pathway to screen for LS found it was cost-effective to screen for LS in those aged $<70$ years. ${ }^{27} \mathrm{~A}$ second study found using MSI, in conjunction with IHC, to be costeffective across different age restrictions. ${ }^{25}$ However, when we investigated MSI in our preliminary analyses, we found its inclusion was more costly than the alternative strategies for all age cohorts with limited benefit (results not shown). Analyses of universal screening using the MLH1-Pathway have indicated that while this strategy is cost-effective, it was not as cost-effective as possible alternatives, with one study determining it was more cost-effective to include both $M L H 1$ methylation and BRAF V600E testing after IHC, ${ }^{29}$ and the other finding cost-effectiveness improved when CRC cases were first triaged with the revised Bethesda guidelines. ${ }^{30}$ We have previously noted that implementation of clinical guidelines in routine practice is poor, ${ }^{4,16,17}$ this would likely impact the effectiveness of this strategy, leading to missed opportunities to diagnose LS and reduced cost-effectiveness. While universal screening using $B R A F$-Pathway has also been shown to 
be cost-effective, ${ }^{26,27,29,31}$ two studies indicated that this strategy was not as effective as alternative strategies that included predictive modeling as a first $\operatorname{step}^{26}$ and the inclusion of both $B R A F$ V600E and MLH1 methylation testing. ${ }^{29}$ When we assessed the $B R A F$-Pathway, we found it to be as effective but more expensive than the MLH1-Pathway at all age thresholds. This was due to the increased number of individuals undergoing germline testing in the BRAF-Pathway as BRAF V600E only achieves $\sim 75 \%$ efficiency as a surrogate marker for MLH1-methylated sporadic CRC showing loss of MLH1/PMS2. ${ }^{18}$ Only one other study has made a direct comparison between the two pathways we investigated, and although the authors determined that the $B R A F$ Pathway was more cost-effective than the MLH1-Pathway, the differences were small. ${ }^{29}$

Benefits of an LS screening program are dependent on ensuring all eligible CRC cases are screened and that those detected with MMR-deficiency receive genetic counseling and germline testing. In our analysis, we assumed all eligible cases would undergo appropriate testing; however, we recognize that this may not occur in practice, ${ }^{17,32}$ as individuals may not wish to participate in genetic testing because of, among other things, possible negative psychological impacts (such as anxiety and depression) and concerns over personal information. ${ }^{33}$ Reducing the proportion of MMR-deficient individuals who agree to genetic counseling and subsequently agree to germline testing decreases yield and total cost in all scenarios, while increasing cost per additional LS case detected. Importantly, such reductions lead to more undiagnosed cases of LS and missed opportunities to identify and monitor atrisk relatives. The greatest benefits of LS screening will only be achieved if screening is appropriately implemented and eligible cases have appropriate and informed access to genetic counseling and germline testing.

An important strength of this study is that the model parameters are derived from two large population-based studies for LS testing and our results align with previous estimates of LS in the CRC population. ${ }^{19,34}$ However, despite this, three limitations are of note. Firstly, this analysis only examined testing incident CRC cases with IHC. However, while we acknowledge that MSI testing, either with or without IHC, is an alternative pathway for triaging CRC cases, ${ }^{16,23}$ our preliminary analyses indicated that this pathway was substantially more expensive, and therefore, we excluded it from further investigations.

Secondly, this analysis only considers costs to identify LS in CRC cases and does not take into account the subsequent costs and cost savings of cascade screening and surveillance of at-risk relatives. While predictive genetic testing of at-risk relatives has been shown to be cost saving in Australia, ${ }^{35}$ there is currently no research into the cost-effectiveness of surveillance in LS carriers. Research with similar cost per LS case detected to ours, which also assessed costs and benefits of surveillance in this group found screening for LS in those aged $<50$ gained 43.6 life year $(\$ 7938 / \mathrm{LYG}){ }^{25}$ When screening was expanded to include those aged 51-60 years, a further 118 life years were gained (\$6380 per additional LYG). An additional 44.3 life years were gained when those aged 61-70 years were screened (\$10 648 per additional LYG). This suggests that with our cost per case detected, cascade screening and surveillance of at-risk individuals will be cost-effective at a willingness-to-pay threshold of \$50 000. As much of the benefit in identifying LS relates to gains in life expectancy in this group, ${ }^{36}$ future research should incorporate analyses of these implications and costs.

Finally, data around the costs of laboratory testing for LS have been difficult to obtain, and our costs may not necessarily reflect the range of costs throughout Australia. To account for this, we conducted sensitivity analyses to provide the lower and upper cost estimates.

\section{Conclusions}

Based on our analysis, MLH1 methylation testing as a follow-up for CRCs showing loss of MLH1 protein expression is more cost-effective than BRAF V600E somatic mutation testing in identifying LS cases. An expanded screening program that includes screening CRC cases diagnosed $<70$ years will identify more LS cases at a reasonable cost. Future research into the yield of LS screening in CRC patients $\geq 70$ years and the potential to offset additional costs by identifying at-risk relatives is needed to determine if universal screening is justified.

\section{Acknowledgments}

We thank Dr Benhur Amanuel, Dr Tony Badrick, Dr Karen Carpenter, and Anne Hawkins and Cassandra Nichols.

\section{References}

1 Australian Institute of Health and Welfare. National Bowel Cancer Screening Program: monitoring report 2016. Cancer series no. 98. Cat. no. CAN 97 Canberra: AIHW; 2016 [Available from: http://www.aihw. gov.au/publication-detail/?id=60129555866].

2 Lynch HT, Lynch JF, Attard TA. Diagnosis and management of hereditary colorectal cancer syndromes: Lynch syndrome as a model. CMAJ 2009; 181: 273-80.

3 Stoffel E, Mukherjee B, Raymond VM et al. Calculation of risk of colorectal and endometrial cancer among patients with Lynch syndrome. Gastroenterology 2009; 137: 1621-7.

4 Vasen HF, Blanco I, Aktan-Collan K et al. Revised guidelines for the clinical management of Lynch syndrome (HNPCC): recommendations by a group of European experts. Gut 2013; 62: 812-23.

5 Senter L, Clendenning M, Sotamaa K et al. The clinical phenotype of Lynch syndrome due to germ-line PMS2 mutations. Gastroenterology 2008; 135: 419-28.

6 Dowty JG, Win AK, Buchanan DD et al. Cancer risks for MLH1 and MSH2 mutation carriers. Hum. Mutat. 2013; 34: 490-7.

7 Baglietto L, Lindor NM, Dowty JG et al. Risks of Lynch syndrome cancers for MSH6 mutation carriers. J. Natl. Cancer Inst. 2010; 102: 193-201.

8 Australian Institute of Health and Welfare. Australian Cancer Incidence and Mortality Books: Colorectal (Bowel) Cancer Canberra: AIHW; 2016 [updated 29 Jan 2016. Available from: http://www.aihw. gov.au/acim-books/].

9 Win AK, Young JP, Lindor NM et al. Colorectal and other cancer risks for carriers and noncarriers from families with a DNA mismatch repair gene mutation: a prospective cohort study. J. Clin. Oncol. 2012; 30: $958-64$.

10 Lynch HT, de la Chapelle A. Hereditary colorectal cancer. N. Engl. J. Med. 2003; 348: 919-32.

11 Stoffel EM, Chittenden A. Genetic testing for hereditary colorectal cancer: challenges in identifying, counseling, and managing high-risk patients. Gastroenterology 2010; 139: 1436-41 41 e1. 
12 Vasen HF, van Ballegooijen M, Buskens E et al. A cost-effectiveness analysis of colorectal screening of hereditary nonpolyposis colorectal carcinoma gene carriers. Cancer 1998; 82: 1632-7.

13 Jarvinen $\mathrm{H}$, Aarnio M, Mustonen $\mathrm{H}$ et al. Controlled 15-year trial on screening for colorectal cancer in families with hereditary nonpolyposis colorectal cancer. Gastroenterology 2000; 118: 829-83.

14 Moller P, Seppala T, Bernstein I et al. Cancer incidence and survival in Lynch syndrome patients receiving colonoscopic and gynaecological surveillance: first report from the prospective Lynch syndrome database. Gut 2015.

15 Jarvinen HJ, Renkonen-Sinisalo L, Aktan-Collan K, Peltomaki P, Aaltonen LA, Mecklin JP. Ten years after mutation testing for Lynch syndrome: cancer incidence and outcome in mutation-positive and mutation-negative family members. J. Clin. Oncol. 2009; 27: 4793-7.

16 Palomaki GE, McClain MR, Melillo S, Hampel HL, Thibodeau SN. EGAPP supplementary evidence review: DNA testing strategies aimed at reducing morbidity and mortality from Lynch syndrome. Genet. Med. 2009; 11: 42-65.

17 Schofield L, Grieu F, Amanuel B et al. Population-based screening for Lynch syndrome in Western Australia. Int. J. Cancer 2014; 135: 1085-91.

18 Buchanan DD, Clendenning M, Rosty C et al. Tumor testing to identify Lynch syndrome in two Australian colorectal cancer cohorts. $J$. Gastroenterol. Hepatol. 2017; 32: 427-38.

19 Ward RL, Hicks S, Hawkins NJ. Population-based molecular screening for Lynch syndrome: implications for personalized medicine. J. Clin. Oncol. 2013; 31: 2554-62.

20 Leggett B, Poplawski N, Pachter N, et al. Cancer Council Australia Colorectal Cancer Guidelines Working Party. Clinical practice guidelines for the prevention, early detection and management of colorectal cancer [Internet]. Sydney: Cancer Council Australia; 2017 [updated 11 December 2017; cited 2018 Apr 5]. Available from: https://wiki.cancer.org.au/australiawiki/index.php?oldid=175314.

21 Giardiello FM, Allen JI, Axilbund JE et al. Guidelines on genetic evaluation and management of Lynch syndrome: a consensus statement by the US Multi-society Task Force on colorectal cancer. Am. J. Gastroenterol. 2014; 109: 1159-79.

22 MBS Online. Medicare benefits schedule-item 73336: Australian Government Department of Health; 2017 [Available from: http:// www9.health.gov.au/mbs/fullDisplay.cfm?type $=i$ tem $\&$ q $=73336 \& q \mathrm{t}=$ item\&criteria $=73336]$.

23 Snowsill T, Huxley N, Hoyle M et al. A model-based assessment of the cost-utility of strategies to identify Lynch syndrome in early-onset colorectal cancer patients. BMC Cancer 2015; 15: 313.

24 Gudgeon JM, Belnap TW, Williams JL, Williams MS. Impact of age cutoffs on a Lynch syndrome screening program. J. Oncol. Pract. 2013; 9: 175-9.

25 Leenen CH, Goverde A, de Bekker-Grob EW et al. Cost-effectiveness of routine screening for Lynch syndrome in colorectal cancer patients up to 70 years of age. Genet. Med. 2016.

26 Barzi A, Sadeghi S, Kattan MW, Meropol NJ. Comparative effectiveness of screening strategies for Lynch syndrome. J. Natl. Cancer Inst. 2015; 107.

27 Ladabaum U, Wang G, Terdiman J et al. Strategies to identify the Lynch syndrome among patients with colorectal cancer: a costeffectiveness analysis. Ann. Intern. Med. 2011; 155: 69-79.

28 Hampel H, Frankel WL, Martin E et al. Feasibility of screening for Lynch syndrome among patients with colorectal cancer. J. Clin. Oncol. 2008; 26: 5783-8.
29 Gudgeon JM, Williams JL, Burt RW, Samowitz WS, Snow GL, Williams MS. Lynch syndrome screening implementation: business analysis by a healthcare system. Am. J. Manag. Care 2011; 17: e288-e300.

30 Pérez-Carbonell L, Guarinos C, Rodríguez Soler M et al. Comparison between universal immunohistochemistry for mismatch repair proteins versus revised Bethesda guidelines in the detection of patients with Lynch syndrome. Gastroenterology 2011; 140: S-97.

31 Mvundura M, Grosse SD, Hampel H, Palomaki GE. The costeffectiveness of genetic testing strategies for Lynch syndrome among newly diagnosed patients with colorectal cancer. Genet. Med. 2010: 93-104.

32 Patel SG, Ahnen DJ, Kinney AY et al. Knowledge and uptake of genetic counseling and colonoscopic screening among individuals at increased risk for Lynch syndrome and their endoscopists from the Family Health Promotion Project. Am. J. Gastroenterol 2016; 111: 285-93.

33 Nicholls SG, Wilson BJ, Craigie SM et al. Public attitudes towards genomic risk profiling as a component of routine population screening. Genome 2013; 56: 626-33.

34 Schofield L, Watson N, Grieu F et al. Population-based detection of Lynch syndrome in young colorectal cancer patients using microsatellite instability as the initial test. Int. J. Cancer 2009; 124: 1097-102.

35 Breheny N, Geelhoed E, Goldblatt J, Ee H, O'Leary P. Economic evaluation of the familial cancer programme in Western Australia: predictive genetic testing for familial adenomatous polyposis and hereditary non-polyposis colorectal carcinoma. Community Genet 2006; 9: 98-106.

36 Grosse SD. When is genomic testing cost-effective? Testing for Lynch syndrome in patients with newly-diagnosed colorectal cancer and their relatives. Healthcare (Basel) 2015; 3: 860-78.

\section{Supporting information}

Additional Supporting Information may be found online in the supporting information tab for this article.

Table S1. Yield and costs for each pathway and age restricted scenario per 1,000 individuals diagnosed with colorectal cancer for the sensitivity analyses with a) the lower bound confidence boundary and b) upper bound confidence boundary.

Table S2. Yield and costs for each pathway and age restricted scenario per 1,000 individuals diagnosed with colorectal cancer for the sensitivity analyses using data from Hampel and colleagues.

Table S3. Yield and costs for each pathway and age restricted scenario per 1,000 individuals diagnosed with colorectal cancer for the sensitivity analyses a) when attendance at genetic counselling reduced to $92.5 \%$ and acceptance of genetic testing reduced to $81 \%$ and b) when attendance at genetic counselling reduced to $92.5 \%$ and acceptance of genetic testing reduced to $90 \%$. 